\title{
Feedback stabilisation of switched systems via iterative approximate eigenvector assignment
}

\author{
Hernan Haimovich and Julio H. Braslavsky
}

\begin{abstract}
This paper presents and implements an iterative feedback design algorithm for stabilisation of discrete-time switched systems under arbitrary switching regimes. The algorithm seeks state feedback gains so that the closed-loop switching system admits a common quadratic Lyapunov function (CQLF) and hence is uniformly globally exponentially stable. Although the feedback design problem considered can be solved directly via linear matrix inequalities (LMIs), direct application of LMIs for feedback design does not provide information on closed-loop system structure. In contrast, the feedback matrices computed by the proposed algorithm assign closedloop structure approximating that required to satisfy Liealgebraic conditions that guarantee existence of a CQLF. The main contribution of the paper is to provide, for single-input systems, a numerical implementation of the algorithm based on iterative approximate common eigenvector assignment, and to establish cases where such algorithm is guaranteed to succeed. We include pseudocode and a few numerical examples to illustrate advantages and limitations of the proposed technique.
\end{abstract}

Index Terms-Switched systems, arbitrary switching, solvable Lie algebras, common eigenvector, linear matrix inequalities, simultaneous triangularisation.

\section{INTRODUCTION}

We consider the discrete-time switching system (DTSS)

$$
x_{k+1}=A_{i(k)} x_{k}+B_{i(k)} u_{k},
$$

with $x_{k} \in \mathbb{R}^{n}, u_{k} \in \mathbb{R}^{m}$, defined by a switching function

$$
i(k) \in \underline{\mathrm{N}}:=\{1,2, \ldots, \mathrm{N}\}, \quad \text { for all } k,
$$

and a set of controllable subsystem pairs $\left\{\left(A_{i}, B_{i}\right): i \in \underline{\mathrm{N}}\right\}$, where the input matrices $B_{i}, i \in \underline{\mathrm{N}}$, have full column rank.

We address feedback control design of the form

$$
u_{k}=K_{i(k)} x_{k},
$$

(which assumes that at every time instant $k$ the "active" subsystem given by $i(k)$ is known) so that the resulting closed-loop system

$$
\begin{aligned}
x_{k+1} & =A_{i(k)}^{\mathrm{CL}} x_{k}, \quad \text { where } \\
A_{i}^{\mathrm{CL}} & =A_{i}+B_{i} K_{i}, \quad \text { for } i \in \underline{\mathrm{N}},
\end{aligned}
$$

be exponentially stable under arbitrary switching.

H. Haimovich is with CONICET and Laboratorio de Sistemas Dinámicos y Procesamiento de Información, Departamento de Control, Facultad de Cs. Exactas, Ingeniería y Agrimensura, Universidad Nacional de Rosario, Riobamba 245bis, 2000 Rosario, Argentina. h. haimovi ch@gmail . com.

J. H. Braslavsky is with the ARC Centre of Excellence for Complex Dynamic Systems and Control, The University of Newcastle, Callaghan NSW 2308, Australia julio.braslavsky@newcastle.edu.au.

Work partially supported by The University of Newcastle 2010 Near Miss Grant G0900210.
It is well-known that ensuring that the closed-loop matrices $A_{i}^{\mathrm{CL}}$ are stable for each $i \in \underline{\mathrm{N}}$ is necessary but not sufficient to ensure the stability of the DTSS (3)-(4) under arbitrary switching [6]. A necessary and sufficient condition for uniform exponential stability under arbitrary switching is the existence of a common Lyapunov function for each of the component subsystems in (3)-(4) [8]. Such Lyapunov functions, however, will in general have complex level sets, which makes their numerical search difficult [11].

The search for common quadratic Lyapunov functions (CQLF), although restrictive, is appealing, since these functions are important in the stabilisation of linear time-invariant systems such as the component subsystems in (1). The design of feedback matrices $K_{i}$ in (2) so that the DTSS (3)-(4) admits a CQLF may be posed as follows.

Problem 1 Given the matrices $A_{i} \in \mathbb{R}^{n \times n}$ and $B_{i} \in \mathbb{R}^{n \times m}$ for $i \in \mathrm{N}$, find feedback matrices $K_{i} \in \mathbb{R}^{m \times n}$ such that the DTSS closed-loop system (3)-(4) admits a CQLF.

Quadratic Lyapunov functions are amenable to efficient numerical computation using linear matrix inequalities (LMIs). For example, Problem 1 can be solved by finding $X=X^{T}>0$ and $N_{i}$ to satisfy the LMIs

$$
\left[\begin{array}{cc}
X & \left(A_{i} X+B_{i} N_{i}\right)^{T} \\
A_{i} X+B_{i} N_{i} & X
\end{array}\right]>0, \quad i \in \underline{\mathrm{N}},
$$

where the required feedback matrices are given by $K_{i}=$ $N_{i} X^{-1}$ and the CQLF is $V(x)=x^{T} X^{-1} x$ (see for example [1], [10]). An advantage of this approach is that the feasibility of the LMIs (5) is necessary and sufficient for the DTSS considered to admit a CQLF. However, such a blind application of LMIs yields no information on the designed closed-loop system structure. The "transparency and interpretability that was a feature of classical techniques" is thus sacrificed, and "a pressing need remains for analytic tools to support the design of stable switched systems" [14] (see also [11], [7]).

As an alternative to LMI approaches, the authors in [14] propose a pole-placement technique for single-input singleoutput continuous-time switching systems. The approach in [14] seeks to guarantee closed-loop uniform global exponential stability under arbitrary switching by designing controllers that achieve a closed-loop common eigenvector structure. By constraining such eigenstructure and the class of controllers allowed, the approach in [14] simplifies the design process, providing analytically transparent solutions in a restricted but practically important class of systems. 
In a similar vein, the present paper proposes an approach to solve Problem 1 by seeking a specific closed-loop structure. Our approach follows from the previous paper [3], which introduced an iterative algorithm to seek feedback gains that make the set of closed-loop subsystems (3)-(4) satisfy Lie algebraic conditions that guarantee the existence of a CQLF [5]. While such Lie-algebraic conditions are restrictive, since they are not necessary for the existence of a CQLF, they offer a way to understand and exploit fundamental system structure in feedback design for DTSS.

The theoretical results in [3] brought forward the following important points: (i) if the proposed Lie-algebraic feedback design problem is feasible, its solution can be found in an iterative manner (similarly to the way solvability of Liealgebras can be checked for autonomous switched systems) by seeking feedback gains that assign a single common stable eigenvector at each iteration; and (ii) in seeking such common eigenvector, if at any iteration more than one vector can be assigned, it is irrelevant which one is chosen by the procedure. These points motivate the development of numerical implementations, which were not discussed in [3].

The present paper deals with a numerical implementation of the iterative algorithm introduced in [3]. A question of interest in the proposed approach is the lack of robustness of the Lie-algebraic conditions that are sought to satisfy by feedback design. Indeed, it is well-known that these conditions are destroyed by arbitrarily small perturbations to the individual matrices $[5, \S 2.2 .4]$, and thus, there are a priori no guarantees that the algorithm introduced in [3] can find any solution at all in a (necessarily approximate) numerical implementation.

However, suppose that for a given set of systems to be stabilised there exists, in a neighbourhood of the original, a feasible (a priori unknown) set of systems for which the resulting closed-loop systems satisfy the Lie-algebraic conditions that guarantee the existence of a CQLF. Then, by continuity of such CQLF, and if the neighbourhood is sufficiently small, there will also exist a CQLF for the original set of systems, despite the fact that it may be impossible to make them satisfy the Lie-algebraic conditions.

The main contribution of the present paper is to, based on the above argument, derive and mathematically justify, for single-input systems, a specific numerical implementation of the design algorithm proposed in [3] that will succeed not only when the Lie-algebraic conditions are satisfied, but also when they are nearly satisfied. The proposed numerical implementation is based on the solution of an optimisation problem that seeks feedback matrices that will achieve closed-loop systems with an approximate common eigenvector. The existence of a CQLF for the corresponding closed-loop DTSS may be readily checked a posteriori with a set of informed LMIs. This step is necessary, since it is in general a priori unknown if the "exact" problem is feasible in some neighbourhood of the original system data. The algorithm has been implemented in MATLAB ${ }^{\circledR}$, and numerical examples to illustrate its application and discuss its advantages and limitations are provided in Section IV.
In the rest of the paper, the proposed algorithm and its core, a procedure for approximate common eigenvector assignment, are first explained in Section II, followed in Section III by the main theoretical results, which justify the numerical implementation of the proposed algorithm for single-input systems. Section V summarises the paper contributions and gives some concluding remarks. The reader can find the main proofs (Theorems 2 and 3) in [4].

Notation: The real and complex numbers are $\mathbb{R}$ and $\mathbb{C}$, with $j:=\sqrt{-1}$. The conjugate transpose of a matrix $M \in$ $\mathbb{C}^{n \times m}$ is denoted $M^{*}$. The $k$-th column of $M$ is denoted $(M)_{:, k}$, and $\operatorname{Im} M$ is the set $\left\{x \in \mathbb{C}^{n}: x=M y, y \in \mathbb{C}^{m}\right\}$. The Euclidean norm, or the corresponding induced matrix norm, is denoted $\|\cdot\|$, and $\rho(\cdot)$ is spectral radius. The Euclidean distance between a vector $v \in \mathbb{C}^{n}$ and a set $\mathscr{V} \subset \mathbb{C}^{n}$ is $\mathrm{d}(v, \mathscr{V})$.

\section{FeEdBack Control Design}

A sufficient condition for the closed-loop DTSS (3)-(4) to admit a CQLF is given by the following result, which is a minor modification of [13, Theorem 6.18].

Lemma 1 If $\rho\left(A_{i}^{\mathrm{CL}}\right)<1$ for $i \in \underline{\mathrm{N}}$, and $\left\{A_{i}^{\mathrm{CL}}: i \in \underline{\mathrm{N}}\right\}$ generates a solvable Lie algebra, then (3)-(4) admits a CQLF.

In matrix terms, the set $\left\{A_{i}^{\mathrm{CL}}: i \in \underline{\mathrm{N}}\right\}$ generates a solvable Lie algebra whenever there exists a nonsingular matrix $T \in \mathbb{C}^{n \times n}$ such that $T^{-1} A_{i}^{\mathrm{CL}} T$ is upper triangular for $i \in \underline{\mathrm{N}}$ (even if $A_{i}^{\mathrm{CL}}$ have real entries, those of $T$ may be complex [2]).

\section{A. The algorithm}

It was established in [3] that given $A_{i}$ and $B_{i}$, there exist feedback matrices $K_{i}$ that cause the Lie algebra generated by $A_{i}^{\mathrm{CL}}$ to be solvable if and only if such feedback matrices can be computed by an algorithm that performs iterative common eigenvector assignment by feedback. A matrix version of such algorithm is given as pseudocode in Algorithm 1 below.

At every iteration $(\ell=1, \ldots, n)$, Algorithm 1 executes Procedure CEA [see (6)]. This procedure attempts to find feedback matrices $F_{i}^{\ell}$ and a vector $v_{1}^{\ell}$ so that $\left(A_{i}^{\ell}+B_{i}^{\ell} F_{i}^{\ell}\right) v_{1}^{\ell}=$ $\lambda_{i}^{\ell} v_{1}^{\ell}$ and $\left|\lambda_{i}^{\ell}\right|<1$ for $i \in \underline{\mathrm{N}}$; namely, so that $v_{1}^{\ell}$ becomes a common eigenvector of a set of closed-loop matrices with corresponding stable eigenvalues. The parameters $\varepsilon_{c}$ and $\varepsilon_{d}$ given as arguments to Procedure CEA are required for numerical reasons, and will be explained in Section II-B.

If Algorithm 1 terminates successfully and at every iteration Procedure CEA is able to find a vector $v_{1}^{\ell}$ and feedback matrices $F_{i}^{\ell}$ such that $\left(A_{i}^{\ell}+B_{i}^{\ell} F_{i}^{\ell}\right) v_{1}^{\ell}=\lambda_{i}^{\ell} v_{1}^{\ell}$ for $i \in \underline{\mathrm{N}}$, then the matrices $A_{i}^{\mathrm{CL}}$ given by (4), with $K_{i}$ computed by Algorithm 1 , are such that $U^{*} A_{i}^{\mathrm{CL}} U$ are upper triangular and $\lambda_{i}^{\ell}$ is the $\ell$-th main-diagonal entry of $U^{*} A_{i}^{\mathrm{CL}} U$.

Note that a slight modification of Algorithm 1 is required to ensure real matrices $K_{i}$ are computed. While such modification is not given here due to space limitations, the implemented computational version of the algorithm, employed in Section IV, does ensure such condition.

The main result in [3] established that a vector $v_{1}^{\ell}$ and feedback matrices $F_{i}^{\ell}$ such that $\left(A_{i}^{\ell}+B_{i}^{\ell} F_{i}^{\ell}\right) v_{1}^{\ell}=\lambda_{i}^{\ell} v_{1}^{\ell}$ with 


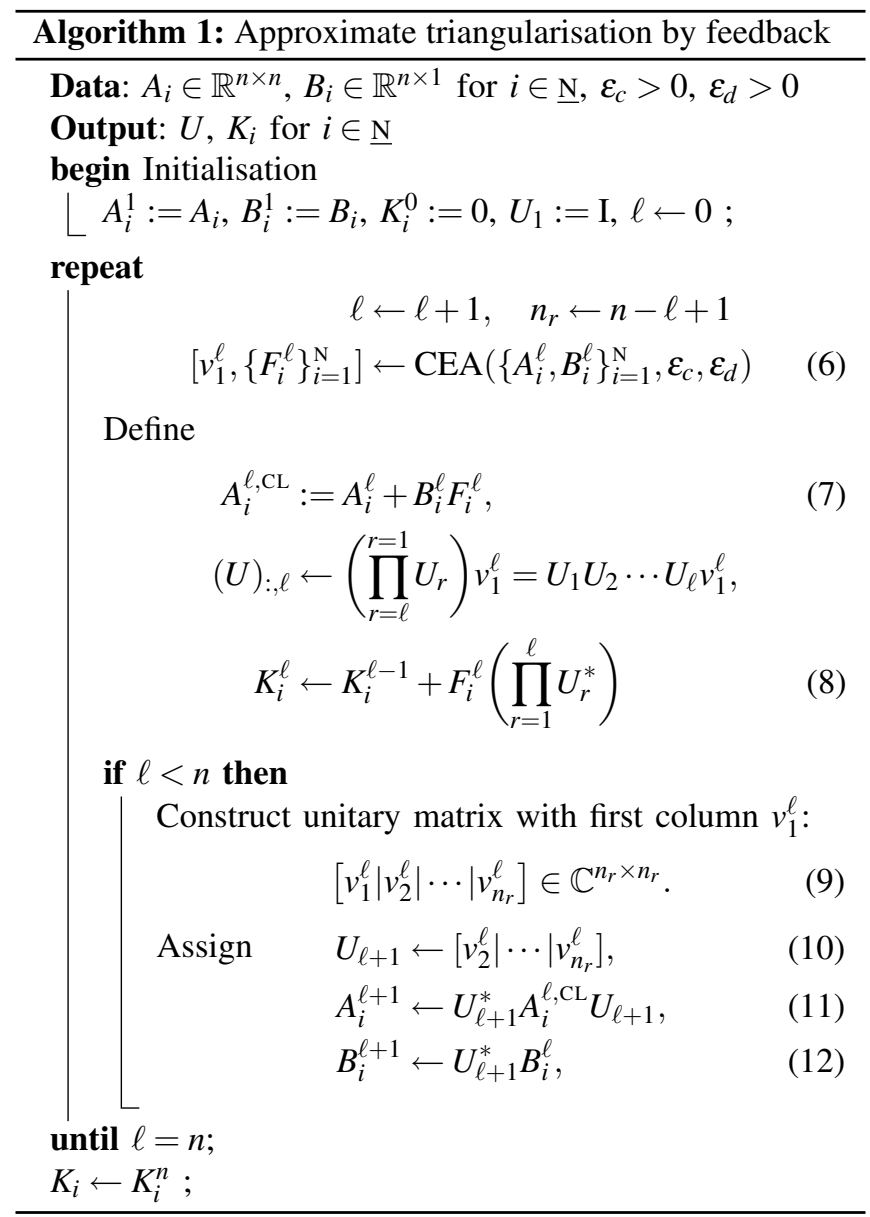

$\left|\lambda_{i}^{\ell}\right|<1$, for $i \in \underline{\mathrm{N}}$, will exist at every iteration of Algorithm 1 if and only if there exist $K_{i}$ such that $A_{i}^{\mathrm{CL}}$ as in (4) generate a solvable Lie algebra and satisfy $\rho\left(A_{i}^{\mathrm{CL}}\right)<1$. Such result was of a theoretical nature, since it is difficult to determine numerically whether a vector is exactly an eigenvector or is close to being one [9].

\section{B. Approximate common eigenvector assignment}

The common eigenvector assignment sought in (6) is the core of Algorithm 1. This section describes a numerical implementation of such common eigenvector assignment procedure, which is given as pseudocode in Procedure CEA. The core of Procedure CEA, in turn, is the optimisation in (13), as we will see after some preliminary definitions.

Note in Procedure CEA that $n_{r}$ represents the state dimension, which decreases by 1 at each iteration of Algorithm 1 . When $n_{r}=1$, the common eigenvector assignment problem is trivially solved if, for $i \in \underline{\mathrm{N}}$, the (scalar) $B_{i}^{\ell}$ are nonzero, since in this case the (scalar) $A_{i}^{\ell, \mathrm{CL}}$ can be arbitrarily chosen.

For the case $n_{r}>1$, Procedure CEA utilises the matrices

$$
\begin{aligned}
E_{i}(v) & :=\left(v v^{*}-\mathrm{I}\right) A_{i}^{\ell}, \\
H_{i}(v) & :=\left(v v^{*}-\mathrm{I}\right) B_{i}^{\ell}, \\
M_{i}(v) & :=-\left(H_{i}(v)^{*} H_{i}(v)\right)^{-1} H_{i}(v)^{*} E_{i}(v), \\
A_{i}^{\ell, \mathrm{CL}}(v) & :=A_{i}^{\ell}+B_{i}^{\ell} M_{i}(v),
\end{aligned}
$$

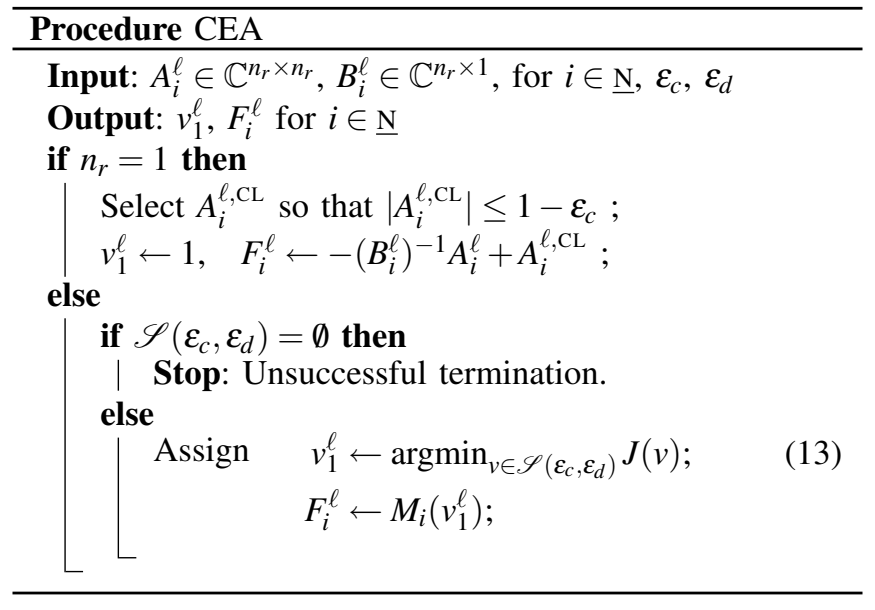

the sets

$$
\begin{aligned}
\mathscr{S}_{1} & :=\left\{v \in \mathbb{C}^{n_{r}}:\|v\|=1\right\} \\
\mathscr{S}_{2}\left(\varepsilon_{c}\right) & :=\bigcap_{i=1}^{\mathrm{N}}\left\{v \in \mathbb{C}^{n_{r}}:\left\|A_{i}^{\ell, \mathrm{CL}}(v) v\right\| \leq 1-\varepsilon_{c}\right\}, \\
\mathscr{S}_{3}\left(\varepsilon_{d}\right) & :=\bigcap_{i=1}^{\mathrm{N}}\left\{v \in \mathbb{C}^{n_{r}}: \mathrm{d}\left(v, \operatorname{Im} B_{i}^{\ell}\right) \geq \varepsilon_{d}\right\}, \\
\mathscr{S}\left(\varepsilon_{c}, \varepsilon_{d}\right) & :=\mathscr{S}_{1} \cap \mathscr{S}_{2}\left(\varepsilon_{c}\right) \cap \mathscr{S}_{3}\left(\varepsilon_{d}\right),
\end{aligned}
$$

and the cost function

$$
J(v):=\sum_{i=1}^{\mathrm{N}}\left\|\left[E_{i}(v)+H_{i}(v) M_{i}(v)\right] v\right\|^{2} .
$$

If $n_{r}>1$, Procedure CEA checks whether the set $\mathscr{S}\left(\varepsilon_{c}, \varepsilon_{d}\right)$ in (21) is empty. The case $\mathscr{S}\left(\varepsilon_{c}, \varepsilon_{d}\right)=\emptyset$ is discussed in Remark 1 below. If $\mathscr{S}\left(\varepsilon_{c}, \varepsilon_{d}\right) \neq \emptyset$, Procedure CEA searches for a vector $v_{1}^{\ell}$ that minimises $J(v)$ as given by (22), subject to the constraint $v \in \mathscr{S}\left(\varepsilon_{c}, \varepsilon_{d}\right)$. The constraint set $\mathscr{S}\left(\varepsilon_{c}, \varepsilon_{d}\right)$ in (21) is the intersection of three sets: $\mathscr{S}_{1}$ in (18), which constrains the search to unit vectors; $\mathscr{S}_{2}\left(\varepsilon_{c}\right)$ in (19), which imposes the stability constraint $\left|\lambda_{i}^{\ell}\right|<1$, as we will shortly demonstrate; and $\mathscr{S}_{3}\left(\varepsilon_{d}\right)$ in (20), which is included for technical reasons discussed next and justified in Section III.

Note that if $\varepsilon_{d}>0$ and $v \in \mathscr{S}_{3}\left(\varepsilon_{d}\right)$, then (20) implies that $v \notin \operatorname{Im} B_{i}^{\ell}$, for $i \in \underline{\mathrm{N}}$. It follows that $H_{i}(v)$ has the same (column) rank as $B_{i}^{\ell}$ [see (15)] and, in this case, $M_{i}(v)$ in (16) is well-defined if $B_{i}^{\ell}$ has full column rank (1.i. columns).

Thus, Procedure CEA requires $B_{i}^{\ell}$ to have full column rank. At the first iteration of Algorithm 1, we have $B_{i}^{1}=$ $B_{i}$ and hence $B_{i}^{1}$ has full column rank by assumption. At subsequent iterations of the algorithm, such condition is ensured by the following result.

Lemma 2 If $v_{1}^{\ell}$ is computed by Procedure CEA using full column rank $B_{i}^{\ell}, i \in \underline{\mathrm{N}}$, then $B_{i}^{\ell+1}$ also have full column rank.

Proof: Since $v_{1}^{\ell}$ is computed by Procedure CEA and $\mathscr{S}\left(\varepsilon_{c}, \varepsilon_{d}\right)$ is compact, then $v_{1}^{\ell} \in \mathscr{S}\left(\varepsilon_{c}, \varepsilon_{d}\right)$ and $v_{1}^{\ell} \notin \operatorname{Im} B_{i}^{\ell}$. The result then follows directly from the fact that the columns of (9) form a basis, (10) and (12). 
We are now ready to see in what sense the minimisation of $J(v)$ is related to the assignment of a common eigenvector.

Lemma 3 Let $\varepsilon_{c}>0, \varepsilon_{d}>0$ and $v \in \mathscr{S}\left(\varepsilon_{c}, \varepsilon_{d}\right)$. Then,

i) $J(v) \geq 0$

ii) $J(v)=0$ if and only if $A_{i}^{\ell, \mathrm{CL}}(v) v=\lambda_{i}^{\ell} v$ with $\left|\lambda_{i}^{\ell}\right|<1$.

iii) There exists $G_{i} \in \mathbb{C}^{m \times n_{r}}$ such that

$$
\left(A_{i}^{\ell}+B_{i}^{\ell} G_{i}\right) v=\lambda_{i}^{\ell} v
$$

if and only if $A_{i}^{\ell, \mathrm{CL}}(v) v=\lambda_{i}^{\ell} v$.

Proof: i) Straightforward from (22) and since $J$ is well defined on $\mathscr{S}\left(\varepsilon_{c}, \varepsilon_{d}\right)$.

ii) $(\Rightarrow) J(v)=0$ implies that

$$
\begin{aligned}
0 & =\left\|\left[E_{i}(v)+H_{i}(v) M_{i}(v)\right] v\right\| \\
& =\left\|\left(v v^{*}-\mathrm{I}\right)\left[A_{i}^{\ell}+B_{i}^{\ell} M_{i}(v)\right] v\right\|,
\end{aligned}
$$

for $i \in \underline{\mathrm{N}}$, where we have used (14), (15) and (22). In turn, (24) implies that

$$
\left[A_{i}^{\ell}+B_{i}^{\ell} M_{i}(v)\right] v=A_{i}^{\ell, \mathrm{CL}}(v) v=\lambda_{i}^{\ell} v
$$

where we have used (17). From (25), $\left|\lambda_{i}^{\ell}\right|=\left\|A_{i}^{\ell, \mathrm{CL}}(v) v\right\| \leq$ $1-\varepsilon_{c}<1$ since $v \in \mathscr{S}\left(\varepsilon_{c}, \varepsilon_{d}\right)$.

$(\Leftarrow)$ Left-multiply (25) by $\left(v v^{*}-\mathrm{I}\right)$ to obtain (24), which implies $J(v)=0$.

iii) $(\Rightarrow)$ Left-multiplying $(23)$ by $\left(v v^{*}-\mathrm{I}\right)$ yields $\left[E_{i}(v)+\right.$ $\left.H_{i}(v) G_{i}\right] v=0$. Let $u_{i}:=G_{i} v$. Then, $E_{i}(v) v=-H_{i}(v) u_{i}$. Since $H_{i}(v)$ has full column rank, then $u_{i}=M_{i}(v) v=G_{i} v$ and hence by (23) $A_{i}^{\ell, \mathrm{CL}}(v) v=\left[A_{i}^{\ell}+B_{i}^{\ell} M_{i}(v)\right] v=\lambda_{i}^{\ell} v$.

$(\Leftarrow)$ Just take $G_{i}=M_{i}(v)$.

Lemma 3 shows that the cost value $J(v)=0$ for some $v$ in $\mathscr{S}\left(\varepsilon_{c}, \varepsilon_{d}\right)$ if and only if such $v$ can be assigned by feedback as a common eigenvector with corresponding stable eigenvalues. Otherwise, $J(v)>0$. Therefore, the search for a vector that minimises $J(v)$ can be interpreted as the search for a vector that is closest to an assignable common eigenvector.

Remark 1 If $\mathscr{S}\left(\varepsilon_{c}, \varepsilon_{d}\right)$ is an empty set, then no feasible vector exists for the optimisation performed by Procedure CEA. Therefore, no vector can be computed, and Procedure CEA and hence Algorithm 1 will terminate unsuccessfully. In the next section, we show that there exist $\varepsilon_{c}, \varepsilon_{d}>0$ that ensure $\mathscr{S}\left(\varepsilon_{c}, \varepsilon_{d}\right)$ is not empty in the cases of interest.

We next establish conditions under which the vector and feedback matrices computed by Procedure CEA cause Algorithm 1 to yield feedback matrices so that the closedloop DTSS admits a CQLF.

\section{MAIN RESUlts}

The results in this section justify the use of Algorithm 1 for feedback control design of single-input systems.

Recall that the constraint set $\mathscr{S}\left(\varepsilon_{c}, \varepsilon_{d}\right)$ in the optimisation (13) solved in Procedure CEA forces the search to be performed over vectors $v$ that are not in $\operatorname{Im} B_{i}^{\ell}$ [by (20)]. The following result justifies this constraint for single-input DTSS with controllable subsystems.
Lemma 4 Let $n_{r}>1, A_{i}^{\ell} \in \mathbb{C}^{n_{r} \times n_{r}}, B_{i}^{\ell} \in \mathbb{C}^{n_{r} \times 1},\left(A_{i}^{\ell}, B_{i}^{\ell}\right)$ be controllable and suppose that $v \neq 0$ and $F_{i}^{\ell}$ satisfy $\left(A_{i}^{\ell}+\right.$ $\left.B_{i}^{\ell} F_{i}^{\ell}\right) v=\lambda_{i} v$, for $i \in \underline{\mathrm{N}}$. Then $v \notin \operatorname{Im} B_{i}^{\ell}$ for $i \in \underline{\mathrm{N}}$.

Proof: Suppose for a contradiction that $v \in \operatorname{Im} B_{k}^{\ell}$ for some $k \in \underline{\mathrm{N}}$. Then $v=B_{k}^{\ell} u$ for some nonzero $u \in \mathbb{C}$. We have $\left(A_{k}^{\ell}+B_{k}^{\ell} F_{k}^{\ell}\right) B_{k}^{\ell} u=\lambda_{k} B_{k}^{\ell} u$ and hence $B_{k}^{\ell}$ and $A_{k}^{\ell} B_{k}^{\ell}$ are linearly dependent, which prevents the pair $\left(A_{k}^{\ell}, B_{k}^{\ell}\right)$ from being controllable because $n_{r}>1$.

Lemma 4 establishes that, for a single-input DTSS with controllable subsystems, every vector that can be made a common eigenvector by feedback will not be contained in the image of any input matrix $B_{i}^{\ell}$. Therefore, in this case such constraint on the optimisation problem is justified.

In the sequel, we will say that a set of matrix pairs is controllable if every matrix pair in the set is controllable. We also require the following definitions.

Definition 1 (CEAS) $A$ set of matrix pairs $\mathscr{Z}^{\ell}=\left\{\left(A_{i}^{\ell} \in\right.\right.$ $\left.\left.\mathbb{C}^{n_{r} \times n_{r}}, B_{i}^{\ell} \in \mathbb{C}^{n_{r} \times 1}\right): i \in \underline{\mathrm{N}}\right\}$ is said to be CEAS (Common Eigenvector Assignable with Stability) if there exist $0 \neq v \in$ $\mathbb{C}^{n_{r}}, \lambda_{i}^{\ell} \in \mathbb{C}$ with $\left|\lambda_{i}^{\ell}\right|<1$, and matrices $F_{i}^{\ell} \in \mathbb{C}^{1 \times n_{r}}$, such that

$$
\left(A_{i}^{\ell}+B_{i}^{\ell} F_{i}^{\ell}\right) v=\lambda_{i}^{\ell} v, \quad \text { for } i \in \underline{\mathrm{N}},
$$

If $\mathscr{Z}^{\ell}$ is CEAS, we say that $v \in \mathbb{C}^{n_{r}}$ and $F_{i} \in \mathbb{C}^{1 \times n_{r}}$ are compatible with $\mathscr{Z}^{\ell}$ whenever (26) holds for $\left|\lambda_{i}^{\ell}\right|<1$.

Definition 2 (SLASF) A set of matrix pairs $\mathscr{Z}=\left\{\left(A_{i} \in\right.\right.$ $\mathbb{C}^{n \times n}, B_{i} \in \mathbb{C}^{n \times 1}$ ) $\left.: i \in \underline{\mathrm{N}}\right\}$ is said to be SLASF (Solvable Lie Algebra with Stability by Feedback) if there exist $K_{i} \in \mathbb{C}^{1 \times n}$ such that $A_{i}^{\mathrm{CL}}$ as in (4) generate a solvable Lie algebra and satisfy $\rho\left(A_{i}^{\mathrm{CL}}\right)<1$. If $\mathscr{Z}$ is $S L A S F$, we say that $K_{i} \in \mathbb{C}^{1 \times n}$ are compatible with $\mathscr{Z}$ if $A_{i}^{\mathrm{CL}}$ as in (4) generate a solvable Lie algebra and satisfy $\rho\left(A_{i}^{\mathrm{CL}}\right)<1$.

The following theorem is a version of the main result in [3] for the specific case of single-input systems.

Theorem 1 Let $\mathscr{Z}=\left\{\left(A_{i} \in \mathbb{C}^{n \times n}, B_{i} \in \mathbb{C}^{n \times 1}\right): i \in \underline{\mathrm{N}}\right\}$, consider Algorithm 1 for some suitable choice of $\varepsilon_{c}$ and $\varepsilon_{d}$, and let $\mathscr{Z}^{\ell}=\left\{\left(A_{i}^{\ell}, B_{i}^{\ell}\right): i \in \underline{\mathrm{N}}\right\}$. Then, $\mathscr{Z}$ is SLASF if and only if $\mathscr{Z}^{\ell}$ is CEAS and $v_{1}^{\ell}, \bar{F}_{i}^{\ell}$ are compatible with $\mathscr{Z}^{\ell}$ for $\ell=1, \ldots, n$.

Suppose that $\hat{\mathcal{Z}}^{\ell}=\left\{\left(\hat{A}_{i}^{\ell}, \hat{B}_{i}^{\ell}\right): i \in \underline{\mathrm{N}}\right\}$ is CEAS. Whenever $\hat{F}_{i}^{\ell}, \hat{v}_{1}^{\ell}$ are compatible with $\hat{\mathscr{Z}}^{\ell}$ and a unitary matrix having $\hat{v}_{1}^{\ell}$ as its first column is given:

$$
\left[\hat{v}_{1}^{\ell}\left|\hat{v}_{2}^{\ell}\right| \cdots \mid \hat{v}_{n-\ell+1}^{\ell}\right],
$$

then $\hat{A}_{i}^{\ell, \mathrm{CL}}, \hat{U}_{\ell+1}, \hat{A}_{i}^{\ell+1}$ and $\hat{B}_{i}^{\ell+1}$ will denote the matrices given by (7), (10), (11) and (12), respectively, when the hatted matrices are employed.

The main result of this section, Theorem 3 below, requires the following preliminary theorem. Due to space limitations, we refer the reader to [4] for the proofs of Theorems 2 and 3. 
Theorem 2 Let $\hat{\mathscr{Z}}^{\ell}=\left\{\left(\hat{A}_{i}^{\ell}, \hat{B}_{i}^{\ell}\right): i \in \mathrm{N}\right\}$ be CEAS and controllable, with $\hat{A}_{i}^{\ell} \in \mathbb{C}^{n_{r} \times n_{r}}, \hat{B}_{i}^{\ell} \in \mathbb{C}^{n_{r} \times 1}, n_{r}>1$. Then, there exist $\varepsilon_{c}^{\star}, \varepsilon_{d}^{\star}>0$ such that each $0<\varepsilon_{c}<\varepsilon_{c}^{\star}$ and $0<\varepsilon_{d}<\varepsilon_{d}^{\star}$ ensure that for every $\varepsilon>0$ there exists a corresponding $\delta>0$ so that for each $A_{i}^{\ell}, B_{i}^{\ell}$ satisfying

$$
\left\{\begin{array}{l}
\left\|\hat{A}_{i}^{\ell}-A_{i}^{\ell}\right\|<\delta, \\
\left\|\hat{B}_{i}^{\ell}-B_{i}^{\ell}\right\|<\delta,
\end{array}\right.
$$

there exist $\hat{v}_{1}^{\ell} \in \hat{\mathscr{S}}\left(\varepsilon_{c} / 2, \varepsilon_{d} / 2\right)$ and $\hat{F}_{i}^{\ell}$ compatible with $\hat{\mathscr{Z}}^{\ell}$ $\left(\hat{v}_{1}^{\ell}, \hat{F}_{i}^{\ell}\right.$ may depend on the specific $\left.A_{i}^{\ell}, B_{i}^{\ell}\right)$, and a unitary matrix (27) that cause

i)

$$
\begin{gathered}
\left\|\hat{v}_{1}^{\ell}-v_{1}^{\ell}\right\|<\varepsilon, \\
\left\|\hat{F}_{i}^{\ell}-F_{i}^{\ell}\right\|<\varepsilon,
\end{gathered}
$$

where $v_{1}^{\ell}$ and $F_{i}^{\ell}$ are the output of Procedure CEA for the specific $A_{i}^{\ell}, B_{i}^{\ell}, \varepsilon_{c}$, and $\varepsilon_{d}$.

ii)

$$
\begin{array}{r}
\left\|\hat{U}_{\ell+1}-U_{\ell+1}\right\|<\varepsilon, \\
\left\|\hat{A}_{i}^{\ell+1}-A_{i}^{\ell+1}\right\|<\varepsilon, \\
\left\|\hat{B}_{i}^{\ell+1}-B_{i}^{\ell+1}\right\|<\varepsilon,
\end{array}
$$

where $U_{\ell+1}, A_{i}^{\ell+1}$ and $B_{i}^{\ell+1}$ are the matrices computed at step $\ell$ of Algorithm 1 from $A_{i}^{\ell}, B_{i}^{\ell}$, $\varepsilon_{c}$ and $\varepsilon_{d}$.

Theorem 2 i) establishes that if the matrices $A_{i}^{\ell}$ and $B_{i}^{\ell}$ given as inputs to Procedure CEA are sufficiently close to some $\hat{A}_{i}^{\ell}$ and $\hat{B}_{i}^{\ell}$ that form a CEAS set $\hat{\mathscr{Z}}^{\ell}$, then the vector $v_{1}^{\ell}$ and feedback matrices $F_{i}^{\ell}$ computed by Procedure CEA will be as close as desired to some $\hat{v}_{1}^{\ell}$ and $\hat{F}_{i}^{\ell}$ compatible with $\hat{\mathcal{Z}}^{\ell}$. In general, whether the (given) matrices $A_{i}^{\ell}, B_{i}^{\ell}$ are sufficiently close to some $\hat{A}_{i}^{\ell}, \hat{B}_{i}^{\ell}$ with the required property will not be known. However, the significance of this result lies precisely in that it establishes a type of continuity relation between the outputs of Procedure CEA and an "exact" result, even if the latter result is not known. Note that such continuity justifies the numerical implementation of the procedure, which will always yield an approximate result. In addition, Theorem 2 ii) shows that if, at step $\ell$ of Algorithm 1, the matrices $A_{i}^{\ell}$ and $B_{i}^{\ell}$ are sufficiently close to some "exact" ones, then the same will happen at step $\ell+1$.

Theorem 3 Let $\hat{\mathscr{Z}}=\left\{\left(\hat{A}_{i} \in \mathbb{C}^{n \times n}, \hat{B}_{i} \in \mathbb{C}^{n \times 1}\right): i \in \underline{\mathrm{N}}\right\}$ be SLASF and controllable. Then, there exist $\varepsilon_{c}^{\star}, \varepsilon_{d}^{\star}>0$ such that each $0<\varepsilon_{c}<\varepsilon_{c}^{\star}$ and $0<\varepsilon_{d}<\varepsilon_{d}^{\star}$ ensure that

i) For every $\varepsilon>0$ there exists a corresponding $\delta>0$ so that for each $A_{i}, B_{i}$ satisfying

$$
\begin{aligned}
& \left\|\hat{A}_{i}-A_{i}\right\|<\delta, \\
& \left\|\hat{B}_{i}-B_{i}\right\|<\delta,
\end{aligned}
$$

there exist $\hat{K}_{i}$ compatible with $\hat{\mathscr{Z}}\left(\hat{K}_{i}\right.$ may depend on the specific $\left.A_{i}, B_{i}\right)$ that cause $\rho\left(\hat{A}_{i}^{\mathrm{CL}}\right) \leq 1-\varepsilon_{c} / 2$ and

$$
\left\|\hat{A}_{i}^{\mathrm{CL}}-A_{i}^{\mathrm{CL}}\right\|<\varepsilon
$$

where $\hat{A}_{i}^{\mathrm{CL}}=\hat{A}_{i}+\hat{B}_{i} \hat{K}_{i}$, and $A_{i}^{\mathrm{CL}}$ from (4) with $K_{i} o b$ tained from Algorithm 1 with inputs $A_{i}, B_{i}, \varepsilon_{c}$, and $\varepsilon_{d}$. ii) There exists $\varepsilon>0$ for which the closed-loop DTSS (3)(4) admits a CQLF, provided (32)-(33) are satisfied with $\delta$ corresponding to $\varepsilon$ as in i) above.

Theorem 3 establishes that Algorithm 1 will compute suitable feedback matrices not only in the "exact" case when the given $A_{i}, B_{i}$ form a SLASF set, but also when they are close to other (possibly unknown) matrices $\hat{A}_{i}, \hat{B}_{i}$ with such property. This justifies the use of Algorithm 1 for control design, since it gives a kind of robustness result for the feedback matrices computed by the algorithm. Theorem 3 also establishes that suitable feedback matrices will be computed for all positive $\varepsilon_{c}$ and $\varepsilon_{d}$ respectively less than $\varepsilon_{c}^{\star}$ and $\varepsilon_{d}^{\star}$. The latter quantities may be not known, since they depend on the possibly unknown $\hat{A}_{i}$ and $\hat{B}_{i}$. Consequently, in theory the parameters $\varepsilon_{c}$ and $\varepsilon_{d}$ should be selected as small as computationally possible. In practice, however, there is a tradeoff in the selection of $\varepsilon_{c}$ since the smaller $\varepsilon_{c}$, the higher the chances of Algorithm 1 yielding unsuitable feedback matrices when more than one common eigenvector could be assigned by feedback with one of them having unstable corresponding eigenvalues and another having stable ones (Procedure CEA could select a local optimiser at the boundary of the constraint set instead of a global optimiser in its interior).

After executing Algorithm 1 to compute feedback matrices $K_{i}$, we can check whether the closed-loop DTSS (3)-(4) admits a CQLF by solving the following LMIs:

$$
P=P^{T}>0, \quad P-\left(A_{i}^{\mathrm{CL}}\right)^{T} P A_{i}^{\mathrm{CL}}>0, \text { for } i \in \underline{\mathrm{N}} .
$$

Note that, in this case, LMIs are used only to check whether a CQLF for the closed-loop system exists, and not for feedback design. If these LMIs are feasible, then not only the closedloop DTSS admits a CQLF but also we have structural information on the DTSS since $K_{i}$ are such that the $A_{i}^{\mathrm{CL}}$ are suitably close to being simultaneously triangularisable.

\section{EXAMPLES}

We next provide some numerical examples to illustrate the advantages and limitations of the proposed feedback design approach. For the numerical implementation of Procedure CEA, a feasible vector is first sought using MATLAB ${ }^{\circledR}$ OptimizATION TOOLBOX ${ }^{\mathrm{TM}}$ function fgoalattain [12]. If such vector is found, it is passed as initial point to the optimisation (13), implemented via the function fmincon.

\section{A. Randomly created DTSS}

The following subsystems were created randomly but such that $\rho\left(A_{1}\right)<1$ and $\rho\left(A_{2}^{-1}\right)<1$.

$$
\begin{aligned}
A_{1} & =\left[\begin{array}{ccc}
0.574 & 0.074 & 0.089 \\
0.074 & 0.572 & -0.091 \\
0.089 & -0.091 & 0.538
\end{array}\right], & B_{1}=\left[\begin{array}{c}
-0.038 \\
0.327 \\
0.175
\end{array}\right], \\
0 & =\left[\begin{array}{ccc}
-0.737 & 0.386 & -1.680 \\
1.351 & 0.638 & 0.035 \\
1.071 & -1.295 & -0.936
\end{array}\right], & B_{2}=\left[\begin{array}{c}
0.114 \\
1.067
\end{array}\right] .
\end{aligned}
$$

Executing Algorithm 1 choosing $\varepsilon_{c}=\varepsilon_{d}=10^{-4}$ yields a feasible optimisation at every iteration and returns

$$
\begin{aligned}
& K_{1}=\left[\begin{array}{lll}
-3.6480 & -7.2304 & 8.7751
\end{array}\right], \\
& K_{2}=\left[\begin{array}{llll}
-0.3159 & 2.0235 & 0.2695
\end{array}\right],
\end{aligned} U=\left[\begin{array}{ccc}
0.4647 & 0.8287 & -0.3120 \\
-0.7770 & 0.2126 & -0.5925 \\
-0.4246 & 0.5178 & 0.7427
\end{array}\right] .
$$


It can be shown that LMIs (35) are feasible and hence the closed-loop DTSS (3)-(4) with $K_{i}$ as in (38) admits a CQLF and is hence stable under arbitrary switching. In this case, it can be checked that $U^{*} A_{i}^{\mathrm{CL}} U$ are not upper triangular but are close in the sense that the entries below the main diagonal are small. Therefore, the use of feedback matrices (38) designed via Algorithm 1 provides some insight into the structure of the closed-loop DTSS.

On the other hand, solving the LMIs (5) for both feedback design and CQLF computation, which yields

$$
K_{1}=\left[\begin{array}{lll}
-1.2267 & -0.7211-1.8731
\end{array}\right], \quad K_{2}=\left[\begin{array}{lll}
-0.5140 & 1.3826 & 1.1613
\end{array}\right],
$$

is guaranteed to produce a closed-loop DTSS stable under arbitrary switching but provides no structural information.

\section{B. DTSS with no CQLF}

Consider the systems

$$
A_{1}=\left[\begin{array}{cc}
0.5 & \alpha \\
0 & 0.5
\end{array}\right], B_{1}=\left[\begin{array}{l}
0 \\
1
\end{array}\right], \quad A_{2}=\left[\begin{array}{cc}
0.5 & 0 \\
\alpha & 0.5
\end{array}\right], B_{2}=\left[\begin{array}{l}
1 \\
0
\end{array}\right] .
$$

For $\alpha=1.5$, the LMIs (5) are not feasible. Therefore, no CQLF exists for this DTSS. Executing Algorithm 1 with $\varepsilon_{c}=\varepsilon_{d}=10^{-4}$ yields an infeasible optimisation at the first iteration: $\mathscr{S}\left(\varepsilon_{c}, \varepsilon_{d}\right)=\emptyset$.

For $\alpha=1.4999$, the LMIs (5) are feasible. In this case, Algorithm 1 for $\varepsilon_{c}=\varepsilon_{d}=10^{-4}$ still yields an infeasible optimisation. However, by reducing $\varepsilon_{c}$ to $10^{-5}$, the algorithm is able to compute suitable stabilising feedback matrices.

\section{CQLF exists but Algorithm 1 fails}

Consider again the DTSS (36)-(37), with the addition of the subsystem

$$
A_{3}=\left[\begin{array}{ccc}
0.352 & 0.159 & -1.129 \\
0.159 & 0 & 0.262 \\
-1.129 & 0.262 & -0.705
\end{array}\right], \quad B_{3}=\left[\begin{array}{c}
-0.433 \\
0 \\
0
\end{array}\right]
$$

Algorithm 1 for $\varepsilon_{c}=\varepsilon_{d}=10^{-4}$ yields

$$
\begin{aligned}
K_{1} & =\left[\begin{array}{lll}
-15.3542 & 3.8969 & -11.3814
\end{array}\right], \\
K_{2} & =\left[\begin{array}{lll}
0.0734 & 0.9747 & 2.7288
\end{array}\right], \\
K_{3} & =\left[\begin{array}{lll}
-1.3542 & 0.8334 & -4.5001
\end{array}\right], \\
U & =\left[\begin{array}{lll}
0.1662+0.0234 j & -0.0918-0.6508 j & -0.7347 \\
0.9744+0.1374 j & 0.0093+0.0662 j & 0.1650 \\
0.0587+0.0083 j & 0.1049+0.7433 j & -0.6580
\end{array}\right] .
\end{aligned}
$$

However, the optimisation informs that there is an active inequality, corresponding to the stability constraint (19). In this case, the LMIs (35) are not feasible and hence no CQLF exists when the feedback matrices (39) are employed.

On the other hand, the LMIs (5) are feasible and hence other feedback matrices may indeed produce a closed-loop DTSS with a CQLF.

\section{CONCLUSIONS}

This paper complements the theoretical results in [3], and contributes to furthering the understanding of fundamental system structure in feedback stabilisation of DTSS. We have presented a numerical implementation of a feedback design approach for DTSS based on Lie-algebraic solvability. The proposed approach seeks feedback matrices to achieve a closed-loop system structure that approximates that required to satisfy such Lie-algebraic stability criteria.

The main theoretical contribution of the paper establishes that if a system for which the Lie-algebraic conditions considered exists in a suitably small neighbourhood of the given system data, then the proposed implementation will find feedback matrices so that the corresponding closed-loop DTSS admits a CQLF even if the considered Lie-algebraic conditions are not met by the given system. However, since the existence of such feasible "exact" system is in general unknown, the resulting closed-loop system is not guaranteed to admit a CQLF, but the latter may be checked with a set of informed LMIs built with the computed closed-loop matrices. Whether such "exact" system exists suitably close to a given system has not been discussed, and remains a topic for further research. Future work will also consider extensions to multiple input systems, which are nontrivial and will possibly require the consideration of controllability indices in the algorithm.

\section{REFERENCES}

[1] J. Daafouz, P. Riedinger, and C. Iung, "Stability analysis and control synthesis for switched systems: A switched Lyapunov function approach," IEEE Transactions on Automatic Control, vol. 47, no. 11, pp. 1883-1887, 2002.

[2] K. Erdmann and M. Wildon, Introduction to Lie algebras. SpringerVerlag London, 2006.

[3] H. Haimovich, J. H. Braslavsky, and F. Felicioni, "On feedback stabilisation of switched discrete-time systems via Lie-algebraic techniques," in Proceedings of the Joint 48th IEEE Conference on Decision and Control and 28th Chinese Control Conference, Shanghai, China, December 2009, pp. 1118-1123, doi:10.1109/CDC.2009.5399527. Also submitted to the IEEE Trans. on Automatic Control.

[4] H. Haimovich and J. Braslavsky, "Feedback stabilisation of switched systems via iterative approximate eigenvector assignment," ArXiv e-prints, September 2010, arXiv:1009.2032v1 [cs.SY]. [Online]. Available: http://arxiv.org/abs/1009.2032v1

[5] D. Liberzon, Switching in systems and control. Birkhäuser, 2003.

[6] D. Liberzon and A. S. Morse, "Basic problems in stability and design of switched systems," IEEE Control Systems Magazine, vol. 19, no. 5, pp. 59-70, 1999.

[7] H. Lin and P. J. Antsaklis, "Stability and stabilisability of switched linear systems: a survey of recent results," IEEE Trans. on Automatic Control, vol. 54, no. 2, pp. 308-322, February 2009.

[8] A. P. Molchanov and Y. S. Pyatnitskiy, "Criteria of asymptotic stability of differential and difference inclusions encountered in control theory," Systems and Control Letters, vol. 13, no. 1, pp. 59-64, 1989.

[9] Y. Mori, T. Mori, and Y. Kuroe, "A solution to the common Lyapunov function problem for continuous-time systems," in Proc. 36th IEEE Conf. on Decision and Control, San Diego, CA, USA, 1997, pp. 35303531.

[10] A. Sala, "Computer control under time-varying sampling period: an LMI gridding approach," Automatica, vol. 41, no. 12, pp. 2077-2082, 2005.

[11] R. Shorten, F. Wirth, O. Mason, K. Wulff, and C. King, "Stability criteria for switched and hybrid systems," SIAM Review, vol. 49, no. 4, pp. 545-592, 2007.

[12] Optimization Toolbox ${ }^{T M}$ User's Guide, 5th ed., The MathWorks Inc., 3 Apple Hill Drive Natick, MA, USA, 2010.

[13] J. Theys, "Joint spectral radius: theory and approximations," Ph.D. dissertation, Center for Systems Engineering and Applied Mechanics, Université Catholique de Louvain, 2005.

[14] K. Wulff, F. Wirth, and R. Shorten, "A control design method for a class of switched linear systems," Automatica, vol. 45, no. 11, pp. 2592-2596, 2009. 